\title{
Probabilistic Map Building Considering Sensor Visibility For Mobile Robot
}

\author{
Kazuma Haraguchi, Nobutaka Shimada, Yoshiaki Shirai and Jun Miura
}

\begin{abstract}
This paper describes a method of probabilistic obstacle map building based on Bayesian estimation. Most active or passive obstacle sensors observe only the most frontal objects and any objects behind them are occluded. Since the observation of distant places includes large depth errors, a conventional method, which does not consider the sensor occlusion often, generate erroneous maps. We introduce a probabilistic observation model, which determines the visible objects. We first estimate probabilistic visibility from the current viewpoint by a Markov chain model based on the knowledge of the average sizes of obstacles and free areas. Then the likelihood of the observations based on the probabilistic visibility are estimated and then the posterior probability of each map grid are updated by Bayesian update rule. Experimental results show that more precise map building can be built by this method.
\end{abstract}

\section{INTRODUCTION}

A mobile robot should know its current position and obstacles and free spaces for self-navigation [1], [2], [3]. This paper deals with the problem of the map building for obstacles and free spaces.

This problem has two important topics: how to represent the map and how to deal with observation errors.

For map representations, there are two typical methods [4]:

1) The map is expressed by the property of obstacles.

2) The map is divided into grids and each grid is assigned probability of obstacle existence.

The former representation requires feature identification and matching and the feature position is update when new observation is available[5]. Latter representation identifies the map grid from which the observation comes. Since laser range sensors, ultrasonic sensors and stereo image sensors can precisely control the direction of transmitting and receiving light or sound, the place from which observation comes is easy to identify. Thus we use the latter map representation here.

For observation errors, we have to consider that stereo method includes depth errors caused by the quantization error and ghost observations caused by correspondence error. Therefore the map building should be established in a probabilistic way based on an error distribution model.

Here we use a probabilistic occupancy grid map for the map representation, which stores the obstacle existence probability in each map grid. In this representation, the obstacle existence probability of each grid is updated by evaluating the likelihood of obtained observation for the grid and integrates it with its prior probability in the way of Bayesian estimation. While Markov Random Field (MRF)

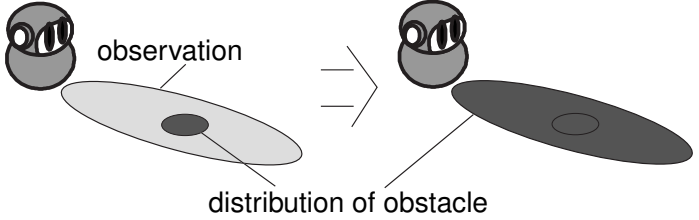

Fig. 1. Result of update when the robot gets the observation of the obstacle of substantial margin of error

[6] solves a generic solution of this map building formulation, its computational cost is huge, especially in case of high grid resolution. Previous methods [7], [8], [9] use the following assumptions for reduction of computation:

1) Observation obtained for each map grid is probabilistically independent (it depends on only the state (obstacle existence) of that grid).

2) Obstacle existence of each map grid is independent to each other.

Most obstacle sensors observe the most frontal objects and any objects behind them are occluded. Since such sensors having sensor occlusion characteristic does not satisfy the above assumption 1, the following serious problems are caused by forcedly using the assumption.

As shown in the left side picture of Fig.1, suppose that dark gray ellipsoidal region has higher probability of obstacle existence than the outside. Then suppose that the obstacle observation with large error (ex. distant observation with stereo vision) is obtained shown as the light gray region in the figure. In that situation, that observation probably comes from the most frontal part of the dark gray region. The assumption 1, however, the obstacle existence probability of each map grid is independently updated by integrating the observation and it is obviously overestimated. As a result everywhere distant from the current robot position tends to be estimated as obstacle in the map. Therefore the assumption 1 should be rejected.

In addition, the assumption 2 also leads to the other problems. If the obstacle existence in each map grid is independent, the probability of that a certain area is open as free-space is estimated as the product of the probability of each map grid. This leads to an obviously irrational result that more precise grid resolution is adopted, abruptly smaller becomes the free-space probability of the same area (in other words, the viewing field is more invisible due to occluding obstacles).

In real scenes, obstacles and free-spaces have a certain size. This points out the existence of co-occurrence between 
the adjacent grids, called spatial continuity. Since the cooccurrence becomes larger when the grid resolution is more precise, the free-space probability can be correctly estimated regardless of the grid resolution. Thus the assumption 2 also should be rejected.

Our map building method correctly considers sensor occlusion and spatial continuity. A certain obstacle is visible if and only if the space between the sensor and the obstacle is entirely open as free-space. Therefore we introduce a novel method of estimating visibility of the obstacle on each map grid by considering spatial continuity, and updating the obstacle existence probability with proper consideration of sensor occlusion.

\section{MAP BUILDING CONSIDERING SENSOR VISIBILITY}

\section{A. Joint Probability Of Adjacent Grids On Each Viewing} Ray

We first divide the map grids into multiple viewing rays. The sensor observes the existence of obstacle on each ray. In this paper, we represent the viewing ray as the 4-connected digit line as shown in Fig.2. On each ray we consider the probabilistic grid state (whether obstacle exists on the grid or not) as a simple Markov chain. Thus each grid state can be represented as the joint probability of two grid states adjacent on each ray.

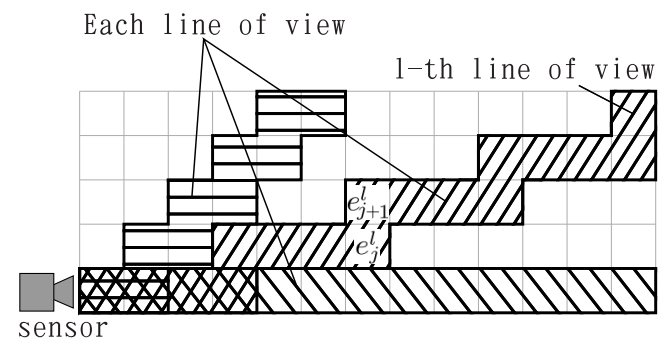

Fig. 2. Approximated Lines of view

We first estimate the joint probability of the $j$ th and $j+1$ th grid (the $j$ th grid is nearer to the sensor) on a viewing ray (see Fig.2). Let $e_{j} \in\left\{E_{j}, \bar{E}_{j}\right\}$ be the state of the $j$ th grid on the ray (E:occupied by an obstacle, $\bar{E}$ : not occupied), and $P\left(e_{j}, e_{j+1} \mid \mathbf{O}\right)$ be the joint probability of the $j$ th and $j+1$ th grid under $\mathbf{O}$, the series of the previous observations. Then the joint probability after the latest observation o obtained is calculated as:

$$
P\left(e_{j}, e_{j+1} \mid o, \mathbf{O}\right)=\alpha P\left(o \mid e_{j}, e_{j+1}, \mathbf{O}\right) P\left(e_{j}, e_{j+1} \mid \mathbf{O}\right) .
$$

$o, \alpha, P\left(e_{j}, e_{j+1} \mid \mathbf{O}\right)$ and $P\left(o \mid e_{j}, e_{j+1}, \mathbf{O}\right)$ respectively denote the observation on the ray, a normalizing constant, the prior probability and the likelihood. The likelihood $P\left(o \mid e_{j}, e_{j+1}, \mathbf{O}\right)$ should be calculated by considering the sensor visibility as the following section.

\section{B. Likelihood considering sensor visibility}

Since an observation, $o$, depends on grid states just on the ray, the likelihood $P\left(o \mid e_{j}, e_{j+1}, \mathbf{O}\right)$ is calculated as:

$$
P\left(o \mid e_{j}, e_{j+1}, \mathbf{O}\right)=\sum_{\mathbf{m} \in \Omega} P\left(o \mid \mathbf{m}, e_{j}, e_{j+1}, \mathbf{O}\right) P\left(\mathbf{m} \mid e_{j}, e_{j+1}, \mathbf{O}\right)
$$

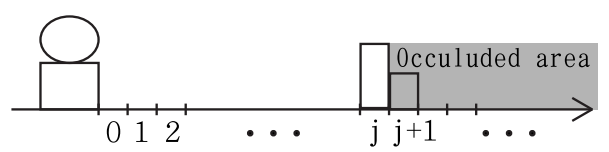

Fig. 3. Occluded area behind the $\mathrm{j}$-th grid

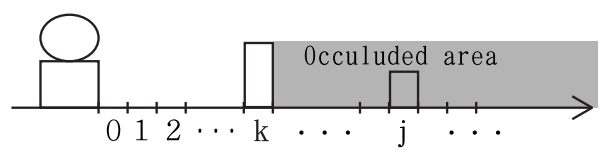

Fig. 4. Occluded area behind the k-th grid

where $\mathbf{m}$ denotes grid states on the ray except $e_{j}, e_{j+1}$. The direct calculation of Eq. 2 requires huge summation of $2^{N_{l}}$ order, where $N_{l}$ denotes the number of grids.

Actually this calculation is drastically reduced by considering the sensor visibility. There exist four cases of the adjacent grid states: $\left(E_{j}, E_{j+1}\right),\left(E_{j}, \bar{E}_{j+1}\right),\left(\bar{E}_{j}, E_{j+1}\right)$, $\left(\bar{E}_{j}, \bar{E}_{j+1}\right)$. In $\left(E_{j}, E_{j+1}\right)$ case (this means both the grid $j$ and $j+1$ are occupied), since grid $j$ occludes $j+1$ as shown in Fig.3, the likelihood $P\left(o \mid E_{j}, E_{j+1}, \mathbf{O}\right)$ is no longer dependent on $j+1$, and then it is represented as:

$$
P\left(o \mid E_{j}, E_{j+1}, \mathbf{O}\right)=P\left(o \mid E_{j}, \mathbf{O}\right)
$$

The above likelihood is acceptable only when grid $j$ is visible from the sensor, namely whole grids between the sensor and grid $j$ are empty. If not so, the most frontal occupied grid $k(<j)$ is observed (see Fig.4). Here, define an stochastic event $F_{k}$ as follows:

$$
F_{k}=\left\{\begin{array}{lr}
E_{0} & (k=0) \\
\bar{E}_{0} \cap \bar{E}_{1} \cap \cdots \cap \bar{E}_{k-1} \cap E_{k}(k>0) .
\end{array}\right.
$$

Since $F_{0}, \cdots, F_{k}$ are mutually exclusive events, the right-hand of Eq.3 is expanded as follows:

$$
\begin{aligned}
P\left(o \mid E_{j}, \mathbf{O}\right) & =\sum_{k=0}^{j} P\left(o \mid F_{k}, E_{j}, \mathbf{O}\right) P\left(F_{k} \mid E_{j}, \mathbf{O}\right) \\
& =\sum_{k=0}^{j} P\left(o \mid F_{k}\right) P\left(F_{k} \mid E_{j}, \mathbf{O}\right)
\end{aligned}
$$

because $o$ is no longer dependent on any grid behind grid $k$ nor the previous observations O. $P\left(o \mid F_{k}\right)$ in Eq.(4) is a sensor kernel model which defines the measurement error distribution of the sensor observation. $P\left(F_{k} \mid E_{j}, \mathbf{O}\right)$ plays a role of the weight for sensor kernel model which decrease the effect of erroneous observations.

$$
\begin{aligned}
& P\left(F_{k} \mid E_{j}, \mathbf{O}\right) \text { in Eq.(4) is expressed as: } \\
& P\left(F_{k} \mid E_{j}, \mathbf{O}\right)=\sum_{\mathbf{e}_{k+1, j-1} \in \Omega_{k+1, j-1}} P\left(F_{k}, \mathbf{e}_{k+1, j-1} \mid E_{j}, \mathbf{O}\right)
\end{aligned}
$$

where $\mathbf{e}_{k+1, j-1}$ is a state of grids between $k+1$ and $j-1$, and $\Omega_{k+1, j-1}$ is a set of the possible states( the number of state is $2^{j-k-1}$ ). Because this calculation amount is too big, we reduce it using a recurrence equation. 
$P\left(o \mid E_{j}, \mathbf{O}\right)$ is expressed as a recurrence equation:

$$
\begin{aligned}
P\left(o \mid E_{j+1}, \mathbf{O}\right)= & \sum_{k=0}^{j} P\left(o \mid F_{k}\right) P\left(F_{k} \mid E_{j}, \mathbf{O}\right) P\left(E_{j+1} \mid E_{j}, \mathbf{O}\right) \\
& +\sum_{k=0}^{j} P\left(o \mid F_{k}\right) P\left(F_{k} \mid E_{j}, \mathbf{O}\right) P\left(E_{j+1} \mid E_{j}, \mathbf{O}\right) \\
& +P\left(o \mid F_{j+1}\right) P\left(F_{j+1} \mid E_{j+1}, \mathbf{O}\right) \\
= & P\left(o \mid E_{i}, \mathbf{O}\right) P\left(E_{j+1} \mid E_{j}, \mathbf{O}\right)+a_{j} P\left(E_{j+1} \mid E_{j}, \mathbf{O}\right) \\
& +P\left(o \mid F_{j+1}\right) P\left(F_{j+1} \mid E_{j+1}, \mathbf{O}\right)
\end{aligned}
$$

where

$$
a_{j}=\left\{\begin{array}{lr}
\sum_{k=0}^{j} P\left(o \mid F_{k}\right) P\left(F_{k} \mid \bar{E}_{j}, \mathbf{O}\right)(j>0) \\
0 & (j=0)
\end{array} .\right.
$$

Eq.(7) is similarly expressed as:

$$
a_{j+1}=P\left(o \mid E_{j}, \mathbf{O}\right) P\left(E_{j} \mid \bar{E}_{j+1}\right)+a_{j} P\left(\bar{E}_{j} \mid \bar{E}_{j+1}\right) .
$$

Thus $P\left(o \mid E_{j+1}, \mathbf{O}\right)$ and $a_{j+1}$, are calculated from $P\left(o \mid E_{j}, \mathbf{O}\right)$ and $a_{j}$.

$P\left(F_{j+1} \mid E_{j+1}, \mathbf{O}\right)$ in Eqs.(6) is visibility which should be estimated based on joint probability of $e_{j}$ and $e_{j+1}$ obtained in the previous time slice:

$$
\begin{aligned}
P\left(F_{j+1} \mid E_{j+1}, \mathbf{O}\right)= & P\left(\bar{E}_{0} \mid \bar{E}_{1}, \mathbf{O}\right) P\left(\bar{E}_{1} \mid \bar{E}_{2}, \mathbf{O}\right) P\left(\bar{E}_{2} \mid \bar{E}_{3}, \mathbf{O}\right) \\
& \cdots P\left(\bar{E}_{j-1} \mid \bar{E}_{j}, \mathbf{O}\right) P\left(\bar{E}_{j} \mid E_{j+1}, \mathbf{O}\right)
\end{aligned}
$$

where $P\left(e_{q} \mid E_{q+1}, \mathbf{O}\right)$ and $P\left(e_{q} \mid E_{q+1}, \mathbf{O}\right)(0 \leq q)$ are expressed as:

$$
\begin{aligned}
P\left(e_{q} \mid E_{q+1}, \mathbf{O}\right) & =\frac{P\left(e_{q}, E_{q+1} \mid \mathbf{O}\right)}{P\left(E_{q} \mid \mathbf{O}\right)} \\
P\left(e_{q} \mid \bar{E}_{q+1}, \mathbf{O}\right) & =\frac{P\left(e_{q}, \bar{E}_{q+1} \mid \mathbf{O}\right)}{P\left(\bar{E}_{q} \mid \mathbf{O}\right)} \\
P\left(e_{q} \mid \mathbf{O}\right) & =P\left(e_{q}, E_{q+1} \mid \mathbf{O}\right)+P\left(e_{q}, \bar{E}_{q+1} \mid \mathbf{O}\right) .
\end{aligned}
$$

The likelihood for the other three cases, $\left(E_{j}, \bar{E}_{j+1}\right)$, $\left(\bar{E}_{j}, E_{j+1}\right),\left(\bar{E}_{j}, \bar{E}_{j+1}\right)$, are derived from $P\left(o \mid E_{k}, \mathbf{O}\right)(k=$ $j, j+1)$ as follows:

$$
\begin{aligned}
& P\left(o \mid E_{j}, \bar{E}_{j+1}, \mathbf{O}\right)=P\left(o \mid E_{j}, \mathbf{O}\right) \\
& P\left(o \mid \bar{E}_{j}, E_{j+1}, \mathbf{O}\right)=P\left(o \mid E_{j+1}, \mathbf{O}\right)-P\left(o, E_{j+1} \mid E_{j}, \mathbf{O}\right) \\
& P\left(o \mid \bar{E}_{j}, \bar{E}_{j+1}, \mathbf{O}\right)=\frac{P(o \mid \mathbf{O})-P\left(o, E_{j} \mid \mathbf{O}\right)-P\left(o, \bar{E}_{j}, E_{j+1} \mid \mathbf{O}\right)}{P\left(\bar{E}_{j}, \bar{E}_{j+1} \mid \mathbf{O}\right)}
\end{aligned}
$$

where $P(o \mid \mathbf{O})$ is constant in the same viewing ray. $P(o \mid \mathbf{O})$ expressed in terms of sensor kernel model $P\left(o \mid F_{k}\right)$ and the grid visibilities $P\left(F_{k} \mid E_{k}\right)$ as:

$$
\begin{aligned}
P(o \mid \mathbf{O}) & =\sum_{k=0}^{\infty} P\left(o \mid F_{k}\right) P\left(F_{k} \mid \mathbf{O}\right) \\
& =\sum_{k=0}^{\infty} P\left(o \mid F_{k}\right) P\left(F_{k} \mid E_{k}, \mathbf{O}\right) P\left(E_{k} \mid \mathbf{O}\right) .
\end{aligned}
$$

$P\left(o, E_{j+1} \mid E_{j}, \mathbf{O}\right), P\left(o, E_{j} \mid \mathbf{O}\right)$ and $P\left(o, E_{j}, E_{j+1} \mid \mathbf{O}\right)$ are expressed as:

$$
\begin{aligned}
P\left(o, E_{j+1} \mid E_{j}, \mathbf{O}\right) & =P\left(o \mid E_{j}, \mathbf{O}\right) P\left(E_{j+1} \mid E_{j}, \mathbf{O}\right) \\
P\left(o, E_{j} \mid \mathbf{O}\right) & =P\left(o \mid E_{j}, \mathbf{O}\right) P\left(E_{j} \mid \mathbf{O}\right) \\
P\left(o, \bar{E}_{j}, E_{j+1} \mid \mathbf{O}\right) & =P\left(o \mid E_{j}, E_{j+1}, \mathbf{O}\right) P\left(\bar{E}_{j}, E_{j+1} \mid \mathbf{O}\right)
\end{aligned}
$$

\section{Posterior update across viewing rays}

The grid posteriors for each viewing ray, estimated in the way of the previous section, conflict with the posteriors of the adjacent rays, because they actually have interactions across the rays and the independent update of each ray is just an approximation. True posteriors should satisfy the following two constraints: the first one is

$$
\sum_{e_{i}, e_{j} \in\{E, \bar{E}\}} P\left(e_{i}, e_{j}\right) \equiv 1
$$

where grid $i$ and $j$ is adjacent, and the second one is

$$
P\left(e_{i}\right) \equiv P\left(e_{i}, E_{m}\right)+P\left(e_{i}, \bar{E}_{m}\right)
$$

for every grid $m$ adjacent to grid $j$ (see Fig. 5). Therefore we

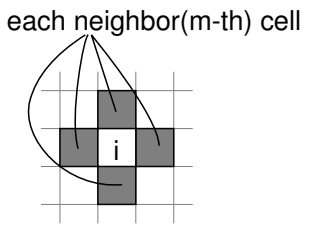

Fig. 5. Adjacent grids

estimate the true posteriors by least squares method under the constraints Eqs.(20),(21). The minimized error $\Delta^{2}$ is written as

$$
\Delta^{2}=\sum_{i, j} \sum_{e_{i}, e_{j}}\left\{P^{*}\left(e_{i}, e_{j}\right)-P\left(e_{i}, e_{j}\right)\right\}^{2}
$$

where $P^{*}\left(e_{i}, e_{j}\right)$ is the conflicting posteriors, $P\left(e_{i}, e_{j}\right)$ is the estimated posteriors satisfying the constraints. This minimization is easily solved by Lagrange's method in low cost.

\section{EXPERIMENTS}

\section{A. Sensor kernel model of stereo vision}

We compared a map building result for the simulation on a viewing ray and a real indoor scene using our method and a conventional method considering no sensor visibility or spatial continuity.

In these experiments, we used edge-based stereo vision for observation of obstacles. Stereo vision provides depth information for each edge feature and it is well-known that the measurement error is inversely proportional to square of the depth. In addition erroneous feature matching can be found in a certain probability. Thus its sensor kernel model $P\left(o \mid F_{k}\right)$, required in Eqs.(4)-(15), are defined here as follows:

$$
P\left(o \mid F_{k}\right)=P(T) P\left(o \mid F_{k}, T\right)+P(\bar{T}) P\left(o \mid F_{k}, \bar{T}\right)
$$

where $o$ is the measured disparity for the viewing ray $l, P(T)$ (fixed to 0.8 in the following experiment, $P(T)+P(\bar{T} \equiv 1)$ ) is the probability of obtaining a correct matching, $P\left(o \mid F_{k}, T\right)$ is a Gaussian (see Fig. 6), $P\left(o \mid F_{k}, \bar{T}\right)$ is a uniform distribution over disparity range $[0,60]$. The obtained sensor kernel model $P\left(o \mid F_{k}\right)$ is shown in Fig.7.

Our method updates the posteriors based on the likelihood considering sensor visibility, Eqs.(4)-(15). 


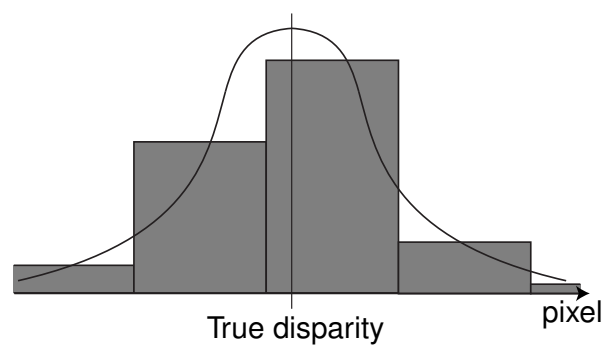

Fig. 6. Probability density of the observation of the disparity

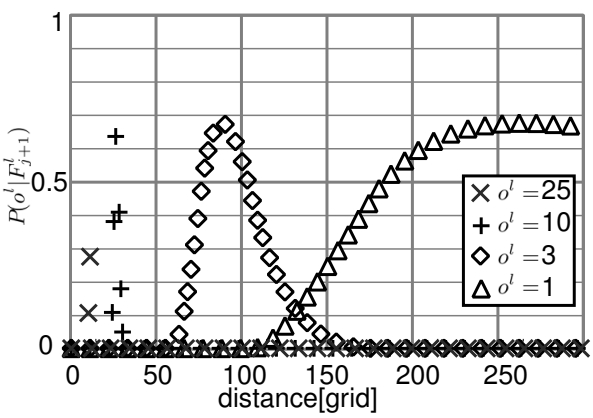

Fig. 7. Observation model

In the conventional method, we assume that state of each grid is independent from state of other grid as:

$$
P\left(e_{i} \mid \mathbf{o}, \mathbf{O}\right)=\frac{P\left(o_{i} \mid e_{i}, \mathbf{O}\right) P\left(e_{i} \mid \mathbf{O}\right)}{P\left(o_{i} \mid E_{i}, \mathbf{O}\right) P\left(E_{i} \mid \mathbf{O}\right)+P\left(o_{i} \mid \bar{E}_{i}, \mathbf{O}\right) P\left(\bar{E}_{i} \mid \mathbf{O}\right)}
$$

where $o_{i}$ denotes the observation on the $i$ th grids and the $l$ th rays. On this assumption we can calculate each grid independently. The following conventional likelihood [7], [8], [9] are adopted as a benchmark:

$$
\begin{aligned}
& P\left(o_{k}=o_{E} \mid E_{k}\right)=P\left(o \mid F_{k}\right) \\
& P\left(o_{k}=o_{E} \mid \bar{E}_{k}\right)=P(\bar{T}) \\
& P\left(o_{k}=o_{\bar{E}} \mid E_{k}\right)=P(\bar{T}) \\
& P\left(o_{k}=o_{\bar{E}} \mid \bar{E}_{k}\right)=P(T)\left\{1-P\left(o \mid F_{k}, T\right)\right\} .
\end{aligned}
$$

\section{B. Posterior update on a viewing ray}

In the following experiments $(B, C$ and $D)$, the initial prior probability $P\left(E_{i}\right)$ is uniformly set to 0.1 and the spatial correlation $c_{q, q+1}$ to 0.871 (used for estimating the spatial continuity). The grid size is $5 \mathrm{~cm} \times 5 \mathrm{~cm}$. These initial parameters are estimated based on average obstacle size $(40 \mathrm{~cm} \times 40 \mathrm{~cm})$ in actual room scene samples.

Since the aim of this paper is to show the effectiveness of our posterior updating considering sensor visibility, we suppose that the exact robot position and orientation is known (NOT SLAM problem).

We compare our method with the conventional one without considering sensor visibility. We use three situations for the map update.
1) Case of prior probability of Uniform distribution: Fig.8 shows the likelihood ratio and the posteriors when we take the observation ( equivalent to the disparity of 10 pixels ) in the uniform distribution. With considering sensor visibility, posteriors in the grid just behind the 30th grid is higher than that without considering visibility because we consider the average size of obstacles( equivalent to $40 \mathrm{~cm}$ ) in this experiment (see section III-A).

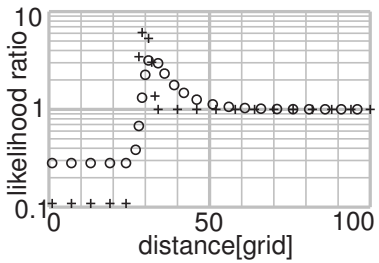

(a) Likelihood

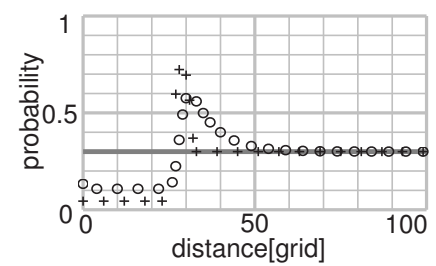

(b) Result of posterior update
Fig. 8. Result of posterior update when the robot get the observation of the obstacle in the uniformly-distributed probability area. "+" represents results of the conventional method. "○" represents results of our method. The gray curve in (b) represents the prior probability.

2) Case of uncertain observation from distant place : In Fig.9 we establish that posteriors are estimated high from the 95th to the 110th grids after we obtain the observations. Fig.9 shows the likelihood and the posteriors when we get the observation ( equivalent to the disparity of 3 pixels) with large error. Without considering visibility, posteriors in the range from the 80th to the 120th grid ( equivalent to the disparity uncertainty of 3 pixels ) becomes high. But we can not obtain the information in the range from the 90th to the 120th grid because we obtain the observation with the error which is larger than distribution of visible obstacles. For this reason with considering visibility, posteriors in the range from the 90th to the 120th grid does not change as Fig.9(b). On the other hand, in front of the range we can obtain the information that the obstacles does not exist. For this reason both methods show that posterior probability of obstacles existence becomes low in front of the range.

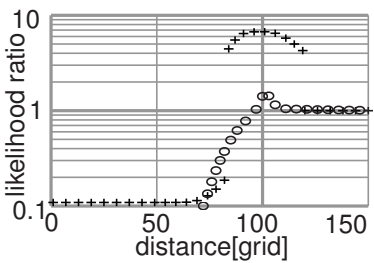

(a) Likelihood

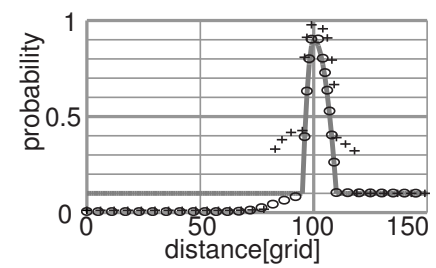

(b) Result of posterior update
Fig. 9. Result of posterior update when the robot get the observation of the obstacle in the uniformly-distributed probability area. "'" represents results of the conventional method. "○" represents results of our method. The gray curve in (b) represents the prior probability. 
3) Case of failure observation for occluded area: In Fig.10 we establish that we obtain the failure observation for an occluded area when existence of obstacle is obvious. The error of this observation is about from the 200th to the 560th grid ( equivalent to the disparity of 1 pixel ). But it is highly possible that the observation is failure because obstacle existence about the 100th grid is already know. With considering sensor visibility, the failure observation is automatically detected and the posteriors kept unchanged.

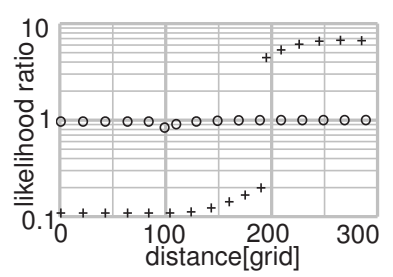

(a) Likelihood

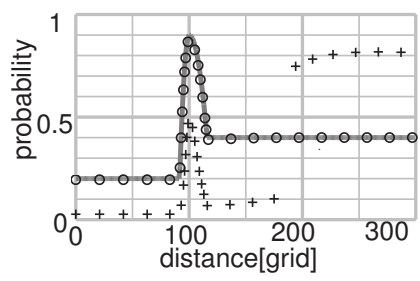

(b) Result of posterior update
Fig. 10. Result of posterior update when the robot get the observation of the obstacle in the uniformly-distributed probability area. "+" represents results of the conventional method. " $\bigcirc$ " represents results of our method. The gray curve in (b) represents the prior probability.

\section{Result of map building for a simulated room}

Next we show the result of map building for a simulated room as shown in Fig.11. We assume mobile robot moved observing obstacles with stereo cameras, starting A point in Fig.11 via B, C, D through the grey line in the figure and finally arrived at B point. By observing obstacles, the robot obtain the disparity with probability of $90 \%$. The probability of the obtained disparity is the same as observation model in Fig.7.

Fig.12 shows the result of conventional method ( without considering occlusion ), and Fig.13 shows the result of our method. They are significantly different at the circular region $\mathrm{X}$ and $\mathrm{Y}$. The conventional method once estimates 'freespace' in X correctly at B. Fig.12(a) shows the state of X ( note that the robot does not observe region $\mathrm{X}$ while moving from $\mathrm{B}$ to $\mathrm{D}$ ).

When the robot observes region $\mathrm{X}$ from farther positions again, it obtains the position of the obstacle behind $\mathrm{X}$ with a large quantization error(Fig.12(b) ). In contrast, our method correctly estimates 'free-space' in $\mathrm{X}$ because the sensor kernel model in the vicinity of the visible obstacle is high weighted and it in the other positions is low weighted.

The conventional method once estimates 'obstacles' in Y correctly when the robot observes the obstacle(Fig.12(a)). Later when the robot observes region Y, it estimates region $\mathrm{Y}$ as 'free-space' due to correspondence error (Fig.12(b) ). In contrast, our method correctly estimates 'obstacles' in Y because the sensor kernel model in the occluded area is less weighted(Fig.13).

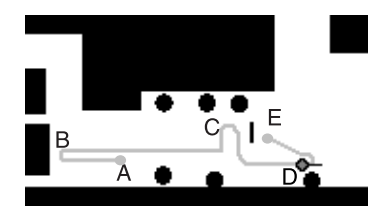

Fig. 11. Truth Of Obstacle Map

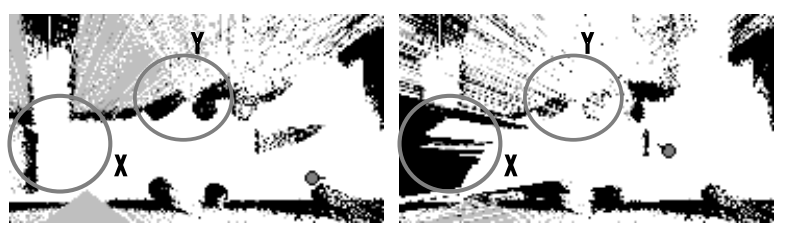
(a) At D
(b) At E

Fig. 12. Result of map update without considering occlusion and spatial dependencies

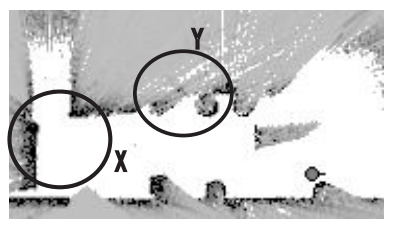

(a) At D

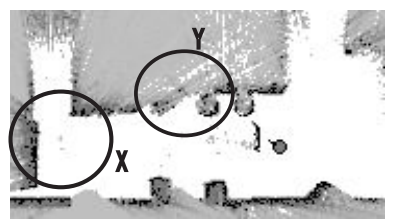

(b) At E
Fig. 13. Result of map update considering occlusion and spatial dependencies

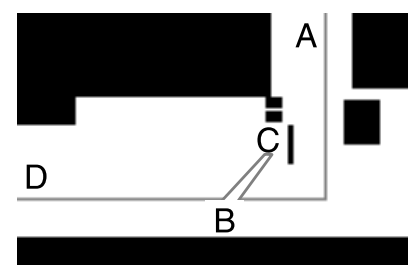

Fig. 14. Rough map of obstacles

\section{Result of map building for an actual room}

Next we show the result of map building for an actual room as shown in Fig. 14. The mobile robot moved in the room observing obstacles with stereo cameras, starting from $A$ point in Fig. 14 via $B, C, B, D$ through the grey line in the figure, and finally arrived at $B$ point.

Fig. 15 shows a view captured by the left camera. Fig.16 and Fig.17 show the result of the convectional method and that of our method respectively. They are significantly different at circular region $\mathrm{X}$. This is caused by a large quantization error just as the previous simulation.

The processing time for one update of all grids is about $1800 \mathrm{~ms}$ on a PC with Athlon X2 4400+ CPU and 2GB memories.

\section{CONCLUSIONS}

We introduced a probabilistic observation model properly considering the sensor visibility. Based on estimating proba- 


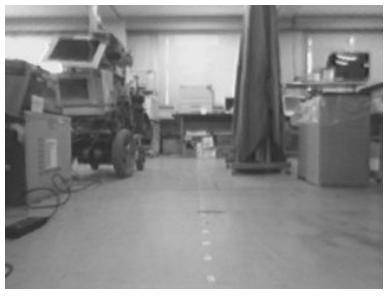

(a) From A in Fig.14

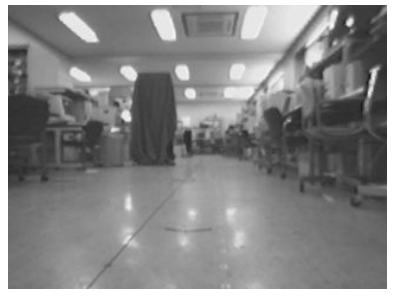

(b) From D in Fig.14
Fig. 15. Robot's view

bilistic visibility of each grid from the current viewpoint, likelihood considering sensor visibility are estimated and then the posteriors of each map grid are updated by Bayesian update rule. For estimating grid visibility, Markov chain calculation based on spatial continuity is employed based on the knowledge of the average sizes of obstacles and free areas.

This paper concentrates the aim on showing the proof of concept of the probabilistic update considering sensor visibility and spatial continuity. For application to the real robot navigation, SLAM framework is necessary. In addition there are moving objects like human and semi-static objects like chairs or doors in the real indoor scene. The expansion to SLAM and environments with movable objects is the future works.

\section{REFERENCES}

[1] M. Montemerlo and S. Thrun, "FastSLAM 2.0: An Improved Particle Filtering Algorithm for Simultaneous Localization and Mapping that Provably Converges," 2002.

[2] S. Thrun, M. Montemerlo, D. Koller, B. Wegbreit, J. Nieto, and E. Nebot, "FastSLAM: An Efficient Solution to the Simultaneous Localization And Mapping Problem with Unknown Data Association," Journal of Machine Learning Research, 2004.

[3] G. Grisetti, C. Stachniss, and W. Burgard, "Improving Grid-based SLAM with Rao-Blackwellized Particle Filters by Adaptive Proposals and Selective Resampling," in Proc. of the IEEE International Conference on Robotics and Automation (ICRA), pp. 2443-2448, 2005.

[4] S. Thrun, "Robotic Mapping: A Survey," in Exploring Artificial Intelligence in the New Millenium (G. Lakemeyer and B. Nebel, eds.), Morgan Kaufmann, 2002.

[5] A. Suppes, F. Suhling, and M. Hötter, "Robust Obstacle Detection from Stereoscopic Image Sequences Using Kalman Filtering," in DAGMSymposium, pp. 385-391, 2001.

[6] Y. Rachlin, J. Dolan, and P. Khosla, "Efficient mapping through exploitation of spatial dependencies," in Proc. of the IEEE/RSJ Int. Conf. on Intelligent Robots and Systems (IROS), pp. 3117-3122, 2005.

[7] J. Miura, Y. Negishi, and Y. Shirai, "Mobile Robot Map Generation by Integrationg Omnidirectional Stereo and Laser Range Finder," in Proc. of the IEEE/RSJ Int. Conf. on Intelligent Robots and Systems (IROS), pp. 250-255, 2002.

[8] S. Thrun, "Learning occupancy grids with forward sensor models," in Autonomous Robots, 2002.

[9] H. P. Moravec, "Sensor fusion in certainty grids for mobile robots," in AI Magazine, Summer, pp. 61-74, 1988.

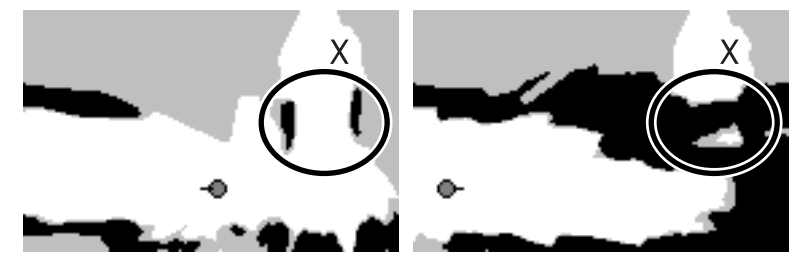

(a) At $\mathrm{B}$ via $\mathrm{A}, \mathrm{B}, \mathrm{C}$

(b) At D via A, B, C, B

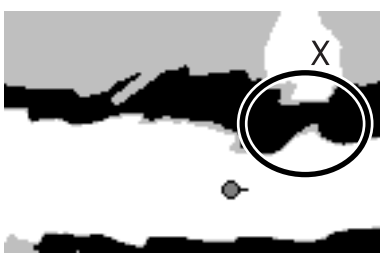

(c) At $\mathrm{B}$ via $\mathrm{A}, \mathrm{B}, \mathrm{C}, \mathrm{B}, \mathrm{D}$

Fig. 16. Result of map update without considering visibility and spatial dependencies

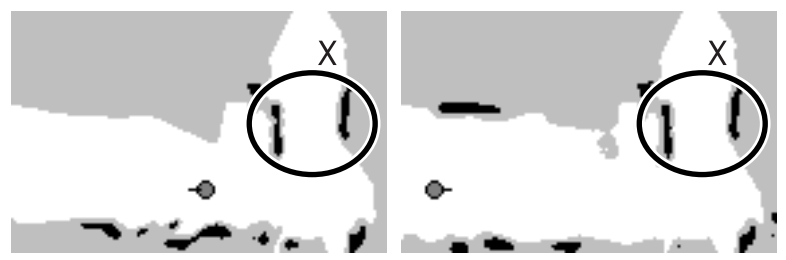

(a) At B via A, B, C

(b) At D via A, B, C, B

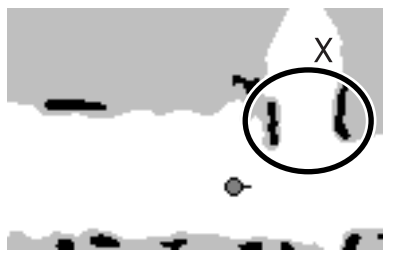

(c) At $\mathrm{B}$ via $\mathrm{A}, \mathrm{B}, \mathrm{C}, \mathrm{B}, \mathrm{D}$

Fig. 17. Result of map update considering visibility and spatial dependencies 